\title{
Amyloid deposits derived from transthyretin in the ligamentum flavum as related to lumbar spinal canal stenosis
}

\author{
Akihiro Yanagisawa ${ }^{1,2}$, Mitsuharu Ueda ${ }^{2}$, Takanao Sueyoshi ${ }^{1}$, Tatsuya Okada ${ }^{1}$, \\ Toru Fujimoto ${ }^{1}$, Yasuhiro Ogi ${ }^{2}$, Keisuke Kitagawa ${ }^{2}$, Masayoshi Tasaki ${ }^{2}$, Yohei Misumi ${ }^{2}$, \\ Toshinori Oshima ${ }^{2}$, Hirofumi Jono ${ }^{3,4}$, Konen Obayashi ${ }^{5}$, Kei Hirakawa ${ }^{6}$, Hitoshi Uchida ${ }^{6}$, \\ Per Westermark ${ }^{7}$, Yukio Ando ${ }^{2}$ and Hiroshi Mizuta ${ }^{1}$ \\ ${ }^{1}$ Department of Orthopaedic Surgery, Graduate School of Medical Sciences, Kumamoto University, \\ Kumamoto, Japan; ${ }^{2}$ Department of Neurology, Graduate School of Medical Sciences, Kumamoto University, \\ Kumamoto, Japan; ${ }^{3}$ Department of Clinical Pharmaceutical Sciences, Graduate School of Pharmaceutical \\ Sciences, Kumamoto University, Kumamoto, Japan; ${ }^{4}$ Department of Pharmacy, Kumamoto University \\ Hospital, Chuo-ku, Kumamoto, Japan; ${ }^{5}$ Department of Morphological and Physiological Sciences, Graduate \\ School of Health Sciences, Kumamoto University, Chuo-ku, Kumamoto, Japan; ${ }^{6}$ Kumamoto Orthopaedic \\ Hospital, Chuo-ku, Kumamoto, Japan and ${ }^{7}$ Department of Immunology, Genetics and Pathology, Uppsala \\ University, Uppsala, Sweden
}

\begin{abstract}
Amyloidosis is a protein conformational disorder with the distinctive feature of extracellular accumulation of amyloid fibrils that come from different proteins. In the ligamentum flavum of the lumbar spine, amyloid deposits were frequently found in elderly patients with lumbar spinal canal stenosis and were at least partially formed by wild-type transthyretin. However, how amyloid deposits in the ligamentum flavum affect lumbar spinal canal stenosis has remained unclear. In this study, we analyzed clinical, pathologic, and radiologic findings of patients with lumbar spinal canal stenosis who had amyloid deposits in the ligamentum flavum. We studied 95 ligamentum flavum specimens obtained from 56 patients with lumbar spinal canal stenosis and 21 ligamentum flavum specimens obtained from 19 patients with lumbar disk herniation. We evaluated histopathologic findings and clinicoradiologic manifestations, such as thickness of the ligamentum flavum and lumbar spinal segmental instability. We found that all 95 ligamentum flavum specimens resected from patients with lumbar spinal canal stenosis had amyloid deposits, which we classified into two types, transthyretin-positive and transthyretinnegative, and that transthyretin amyloid formation in the ligamentum flavum of patients with lumbar spinal canal stenosis was an age-associated phenomenon. The amount of amyloid in the ligamentum flavum was related to clinical manifestations of lumbar spinal canal stenosis, such as thickness of the ligamentum flavum and lumbar spinal segmental instability, in the patients with lumbar spinal canal stenosis with transthyretin-positive amyloid deposits. To our knowledge, this report is the first to show clinicopathologic correlations in transthyretin amyloid deposits of the ligamentum flavum. In conclusion, transthyretin amyloid deposits in the ligamentum flavum may be related to the pathogenesis of lumbar spinal canal stenosis in elderly patients.
\end{abstract}

Modern Pathology (2015) 28, 201-207; doi:10.1038/modpathol.2014.102; published online 5 September 2014

Amyloidosis is a disorder of protein conformation in which extracellular accumulation of amyloid fibrils that derive from different proteins occurs. ${ }^{1,2}$ Thus far, 30 distinct protein precursors of amyloid fibrils were identified as causing different kinds of

Correspondence: Dr M Ueda, MD, PhD, Department of Neurology, Graduate School of Medical Sciences, Kumamoto University, 1-1-1 Honjo, Chuo-ku, Kumamoto 860-0811, Japan.

E-mail: mitt@rb3.so-net.ne.jp

Received 15 April 2014; Received 23 May 2014; accepted 28 May 2014; published online 5 September 2014 amyloid-associated diseases. ${ }^{3}$ In each kind of amyloid deposits, specific features, including aging, mutation, inflammation, and tumors, can effect diseasecausing polymerization of soluble proteins that function in the body. ${ }^{1,2}$ Transthyretin, a serum protein synthesized mainly in the liver, causes two types of systemic amyloidosis. One type is a hereditary systemic amyloidosis (familial amyloid polyneuropathy), which is induced by mutant transthyretin. $^{4-6}$ The other type is senile systemic amyloidosis, which is an age-related sporadic systemic amyloidosis that is induced by wild-type 
transthyretin. ${ }^{7-9}$ Besides full-length wild-type transthyretin, C-terminal wild-type transthyretin fragments beginning at positions 46-52 usually occur in amyloid deposits obtained from patients with senile systemic amyloidosis as well as many cases with hereditary transthyretin amyloidosis. ${ }^{8,10}$

Lumbar spinal canal stenosis is the most common spinal disorder in elderly people. Although hypertrophy of the ligamentum flavum is one of the major factors associated with lumbar spinal canal stenosis, the mechanism of ligamentum flavum hypertrophy is not well understood. Spinal ligaments of elderly patients reportedly often contained amyloid deposits, but precursor proteins of those amyloid fibrils were not clarified. ${ }^{11,12}$ We recently reported that amyloid deposits in the ligamentum flavum were at least partially derived from wild-type transthyretin in elderly lumbar spinal canal stenosis patients. ${ }^{13}$ Another recent paper suggested that transthyretin amyloid formation may be a common cause of lumbar spinal canal stenosis. ${ }^{14}$ We also reported that a senile systemic amyloidosis patient with multifocal spinal transthyretin amyloid deposits developed severe destructive spondylosis and radiculomyelopathy. ${ }^{15}$ However, how amyloid deposits in the ligamentum flavum affect lumbar spinal canal stenosis in which the ligamentum flavum shows degeneration and hypertrophy remains unclear. In this study, we analyzed clinical, pathologic, and radiologic findings of lumbar spinal canal stenosis patients with amyloid deposits. To our knowledge, this report is the first to show clinical-radiological-pathological correlations in transthyretin amyloid deposits in lumbar spinal canal stenosis.

\section{Patients and methods}

\section{Patients}

We used 95 ligamentum flavum specimens obtained from 56 patients with lumbar spinal canal stenosis and 21 ligamentum flavum specimens obtained from 19 patients with lumbar disk herniation; all patients underwent lumbar spinal surgery for in Kumamoto Orthopaedic Hospital from 2012 to 2013.

\section{Histologic Examination}

Tissue samples were fixed in $10 \%$ formalin, embedded in paraffin, serially sectioned at a thickness of $4 \mu \mathrm{m}$, and placed onto microscope slides. Sections were stained with hematoxylin-eosin and alkaline Congo red, after which they were viewed under polarized light to check for green birefringence. The extent of amyloid deposition, determined by measuring the degree of Congo red-positive areas, was ascertained with a computer and the public domain ImageJ program developed by the US National Institutes of Health and available at http://rsb.info.nih.gov/ij/index.html.

\section{Immunohistochemistry}

Deparaffinized sections were incubated in $3 \%$ hydrogen peroxide for 15 min to block endogenous peroxidase. Goat serum (Dako, Glostrup, Denmark) was used to block nonspecific background staining. A rabbit polyclonal anti-transthyretin antibody (Dako) and a horseradish peroxidase-conjugated goat anti-rabbit immunoglobulin antibody (Dako) were used at 1:100 dilution as the first and second antibodies, respectively. Reactivity was visualized by using the DAB Liquid System (Dako). Sections were counterstained with hematoxylin.

\section{Magnetic Resonance Imaging of the Ligamentum Flavum}

The thickness of the ligamentum flavum at the facet joint of the affected spinal level was measured on axial T1-weighted magnetic resonance images. ${ }^{16} \mathrm{We}$ measured the thickness of the ligamentum flavum three times and used the average of the three repeated measurements as the final value.

\section{Radiography of the Lumbar Spine}

Lateral functional flexion-extension radiographs were used to evaluate lumbar spinal segmental instability. ${ }^{17}$ The adjacent vertebral end-plate angle (total degrees) was measured by using the lumbar flexion and extension radiographs at the operative level. ${ }^{18}$

\section{Nephelometry to Measure Serum Transthyretin Concentrations and Mass Spectrometric Analysis of Serum Transthyretin}

Serum transthyretin concentrations were determined via nephelometry in seven serum samples from lumbar spinal canal stenosis patients with transthyretin-positive amyloid deposits and eight serum samples from lumbar spinal canal stenosis patients with transthyretin-negative amyloid deposits. We excluded patients with low albumin concentrations $(<4.1 \mathrm{~g} / \mathrm{dl})$ and/or high C-reactive protein concentrations $(>0.3 \mathrm{mg} / \mathrm{dl})$ in serum. To detect wild-type and mutant transthyretins, serum samples were analyzed by using surface-enhanced laser desorption/ionization time-of-flight mass spectrometry with the Q10 ProteinChip (Bio-Rad, Hercules, CA, USA), a strong anion exchange array with quaternary amine functionality, as described previously. ${ }^{19}$

\section{Detection of Fragmented Transthyretin in Amyloid Deposits}

To extract amyloid-forming proteins from formalinfixed, paraffin-embedded specimens, we used biochemical methods of Layfield et al. ${ }^{20}$ In brief, sodium dodecyl sulfate-polyacrylamide gel electrophoresis was applied to process isolated tissue pellets that 
were incubated in Laemmli sample buffer (Bio-Rad) containing $8 \mathrm{M}$ urea at $90{ }^{\circ} \mathrm{C}$ for $15 \mathrm{~min}$, after which samples were transferred to a nitrocellulose membrane (Bio-Rad) for immunoblotting. A polyclonal antiserum against truncated transthyretin 50-127 (transthyretin 50-127), produced in rabbits, was diluted 1:2000 and used as the primary antibody. ${ }^{21}$ A goat anti-rabbit antibody conjugated with horseradish peroxidase (Dako), diluted 1:5000, was used as the secondary antibody. The reaction was visualized by using an enhanced chemiluminescence system (GE Healthcare, Buckinghamshire, UK).

\section{Statistical Analysis}

Data were evaluated with Student's $t$-test and Fisher's exact test. All analyses were performed with JMP Version 5.1 (SAS Institute Japan, Tokyo, Japan). $P$-values of $<0.05$ were regarded as statistically significant.

\section{Ethics}

The study protocol was approved by Human Ethics Review Committee of Kumamoto University and Kumamoto Orthopaedic Hospital. A signed consent form was obtained from all patients or a family member.

\section{Results}

\section{Amyloid Deposits in the Ligamentum Flavum}

All 95 ligamentum flavum specimens resected from lumbar spinal canal stenosis patients contained amyloid deposits. Immunohistochemistry revealed positive transthyretin staining in amyloid deposits in 43 of 95 specimens (Table 1). The anti-transthyr- etin antibody did not react with amyloid deposits in the other specimens. The amount of amyloid deposited in transthyretin-positive amyloid cases was higher than that in transthyretin-negative amyloid cases. As Figure 1 shows, transthyretin-positive amyloid occurred in massive deposits, whereas transthyretin-negative amyloid formed small spotty deposits. Lumbar spinal canal stenosis patients with transthyretin-positive amyloid deposits had no symptoms of cardiac amyloidosis, which is major symptom of senile systemic amyloidosis. We found no amyloid deposition in blood vessels in 40 $(95.6 \%)$ of 41 specimens containing transthyretinpositive amyloid deposits in the ligamentum flavum (Supplementary Figure S1 and Supplementary Table S1). One case having transthyretin-positive amyloid deposits in blood vessels of the ligamentum flavum did not show any clinical symptoms suggesting senile systemic amyloidosis such as cardiac failure (Supplementary Figure S1 and Supplementary Table S1). We also investigated the presence of amyloid deposits in the ligamentum flavum of lumbar disk herniation patients. Transthyretin-negative amyloid deposits were found 9 (42.9\%) of 21 specimens (Table 2). We found no transthyretin-positive amyloid deposits in the ligamentum flavum of lumbar disk herniation patients.

\section{Relationship Between Transthyretin Amyloid Deposits and Age}

Lumbar spinal canal stenosis patients with transthyretin-positive amyloid deposits were older than patients with transthyretin-negative findings (Table 1). The occurrence of transthyretin-positive amyloid deposits increased with age (Figure 2a), but no relationship existed between the amount of transthyretin amyloid and age (Figure 2b).

Table 1 Characteristics of lumbar spinal canal stenosis patients with transthyretin-positive or transthyretin-negative amyloid deposits in the ligamentum flavum

\begin{tabular}{|c|c|c|c|}
\hline Characteristic & Transthyretin + & Transthyretin - & $\mathrm{P}$-value \\
\hline Frequency of occurrence, $n(\%)$ & $43(45.3 \%)$ & $52(54.7 \%)$ & \\
\hline Males/females, $n$ & $26 / 17$ & $42 / 10$ & \\
\hline Age (years; mean \pm s.d.) & $74 \pm 7.6$ & $68 \pm 6.4$ & $<0.0001$ \\
\hline Amount of amyloid deposited (\%) & $4.34 \pm 3.89$ & $0.26 \pm 0.33$ & $<0.0001$ \\
\hline Mean ligamentum flavum thickness (mm) & $4.36 \pm 0.79$ & $3.39 \pm 0.75$ & $<0.0001$ \\
\hline Lumbar spinal segmental instability (deg) & $8.1 \pm 3.3$ & $5.6 \pm 2.6$ & $<0.0005$ \\
\hline Destructive spondylosis, $n$ & 0 & 0 & \\
\hline Cardiac failure, $n$ & 0 & 0 & \\
\hline \multicolumn{4}{|l|}{ Level of harvested ligamentum flavum, $\mathrm{n}$} \\
\hline $\mathrm{L} 1 / 2$ & 2 & 3 & \\
\hline $\mathrm{L} 2 / 3$ & 6 & 13 & \\
\hline $\mathrm{L} 3 / 4$ & 13 & 15 & \\
\hline $\mathrm{L} 4 / 5$ & 18 & 17 & \\
\hline $\mathrm{L} 5 / \mathrm{S}$ & 4 & 4 & \\
\hline
\end{tabular}

Abbreviations: Transthyretin +, lumbar spinal canal stenosis patients with transthyretin-positive amyloid deposits; transthyretin -, lumbar spinal canal stenosis patients with transthyretin-negative amyloid deposits. 
a

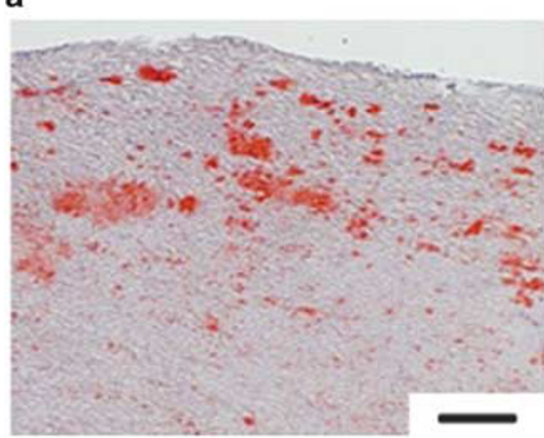

d

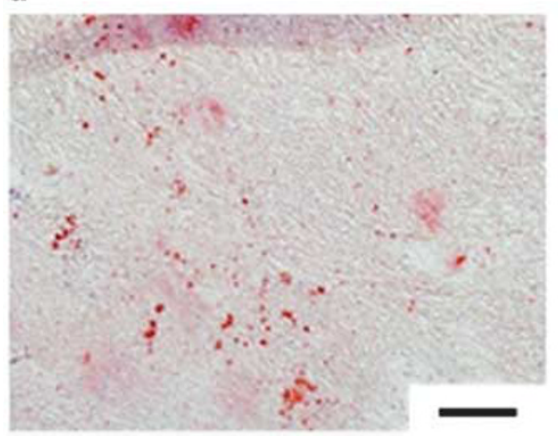

b

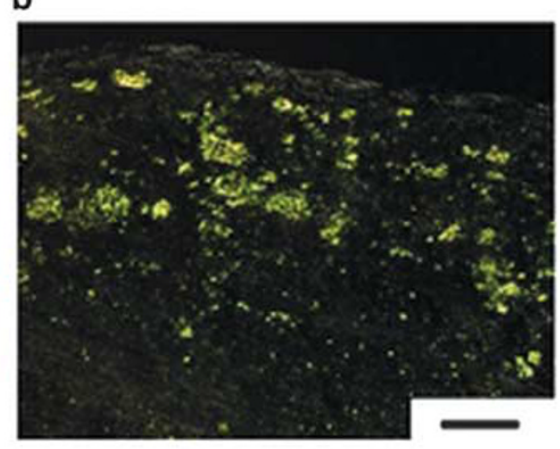

e

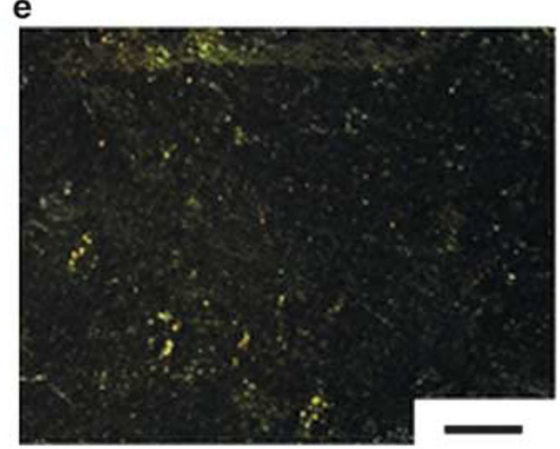

c

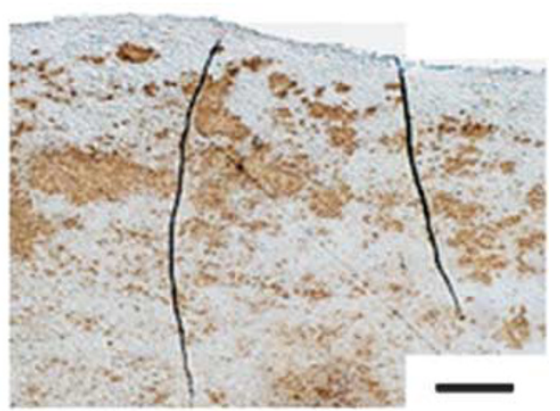

Figure 1 Amyloid deposits in the ligamentum flavum of lumbar spinal canal stenosis patients. (a-c) Transthyretin-positive amyloid deposits in the ligamentum flavum. (d and e) Transthyretin-negative amyloid deposits in the ligamentum flavum. (a and d) Congo red staining. (b and e) The same sections viewed with polarized light. (c) Immunohistochemical staining with an anti-human transthyretin polyclonal antibody (Dako). Scar bars, $200 \mu \mathrm{m}$.

Table 2 Characteristics of lumbar disk herniation patients with or without amyloid deposits in the ligamentum flavum

\begin{tabular}{lcc}
\hline Characteristic & Without amyloid deposits & With amyloid deposits \\
\hline Frequency of occurrence, $n(\%)$ & $12(57.1 \%)$ & $9(42.9 \%)$ \\
Transthyretin-positive amyloid & Not applicable & $0(0 \%)$ \\
Males/females, $n$ & $10 / 2$ & $7 / 2$ \\
Age (years, mean \pm s.d.) & $41.1 \pm 10.6$ & $57.6 \pm 10.1$ \\
Mean ligamentum flavum thickness (mm) & $2.54 \pm 0.38$ & $2.98 \pm 0.27$ \\
Level of harvested ligamentum flavum, $\mathrm{n}$ & & 0 \\
L1/2 & 0 & 2 \\
L2/3 & 0 & 2 \\
L3/4 & 1 & 4 \\
L4/5 & 7 & 2 \\
L5/S & 4 & 0.051 \\
\hline
\end{tabular}

Relationship Between the Amount of Transthyretin Amyloid in the Ligamentum Flavum and Ligamentum Flavum Thickness

Magnetic resonance imaging revealed that the ligamentum flavum of lumbar spinal canal stenosis patients with transthyretin-positive amyloid deposits was thicker than the ligamentum flavum of lumbar spinal canal stenosis patients with transthyretin-negative amyloid deposits (Table 1). The amount of amyloid in the ligamentum flavum was related to ligamentum flavum thickness in lumbar spinal canal stenosis patients with transthyretin amyloid deposits (Figure 3a).

Relationship Between the Amount of Transthyretin Amyloid in the Ligamentum Flavum and Lumbar Spinal Segmental Instability

Lateral functional flexion-extension radiography revealed that the lumbar spinal segmental instability of lumbar spinal canal stenosis patients with transthyretin-positive amyloid deposits was greater 

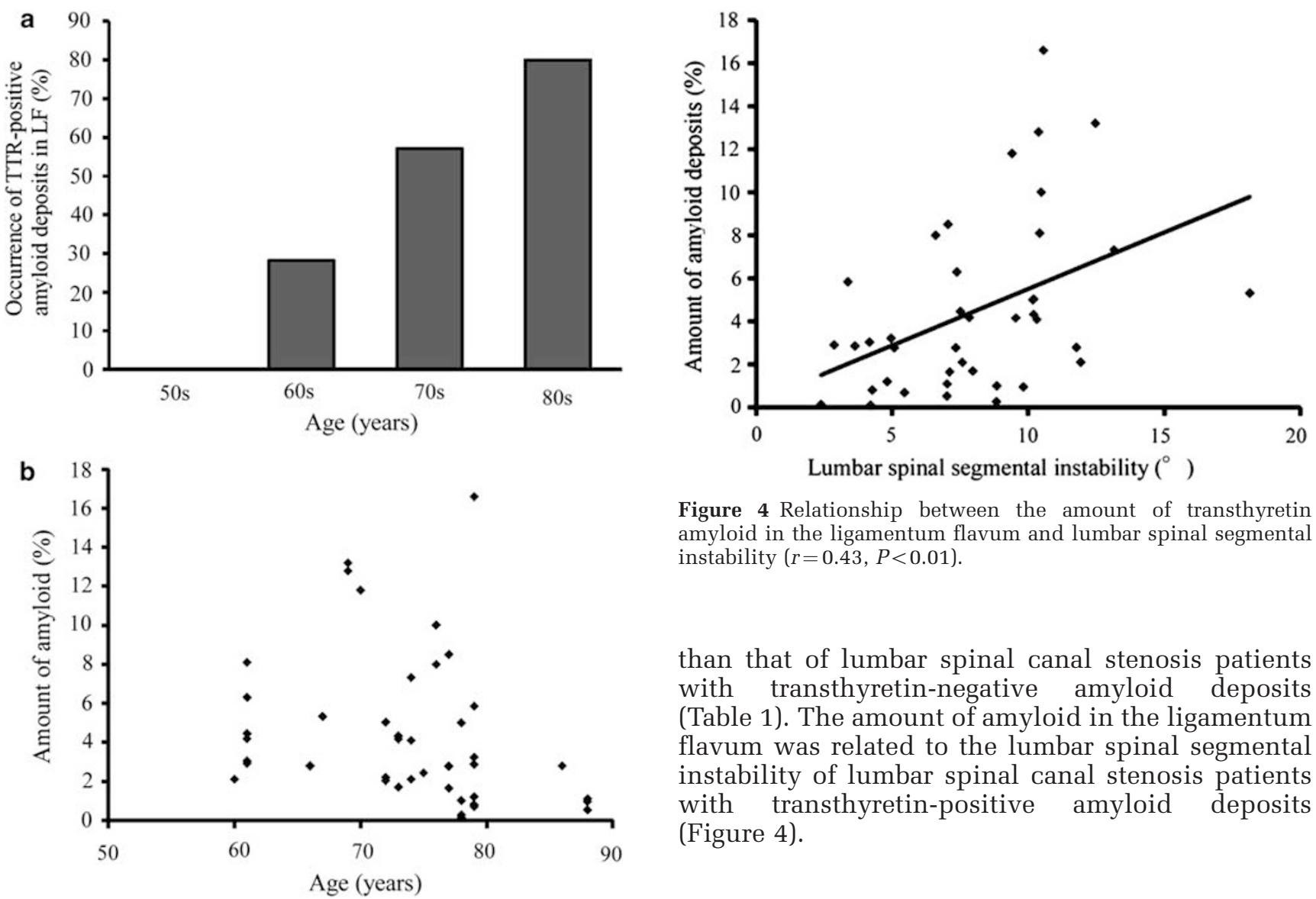

Figure 2 Occurrence and amount of transthyretin amyloid in the ligamentum flavum of lumbar spinal canal stenosis patients. (a) Occurrence of transthyretin-positive amyloid deposits according to age. (b) The amount of transthyretin-positive amyloid according to age $(r=0.25, P=0.1)$.

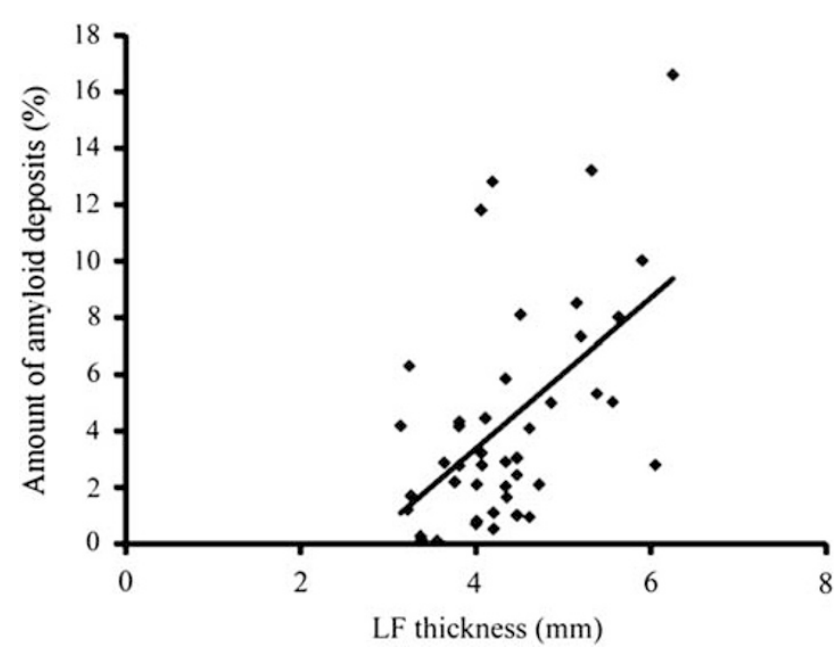

Figure 3 Relationship between the amount of transthyretin amyloid in the ligamentum flavum and ligamentum flavum thickness $(r=0.54, P<0.0005)$.
Figure 4 Relationship between the amount of transthyretin amyloid in the ligamentum flavum and lumbar spinal segmental instability $(r=0.43, P<0.01)$.

than that of lumbar spinal canal stenosis patients with transthyretin-negative amyloid deposits (Table 1). The amount of amyloid in the ligamentum flavum was related to the lumbar spinal segmental instability of lumbar spinal canal stenosis patients with transthyretin-positive amyloid deposits (Figure 4).

\section{Serum Transthyretin Concentrations and Mass Spectrometric Analysis of Serum Transthyretin}

We analyzed serum transthyretin concentrations in lumbar spinal canal stenosis patients with transthyretin-positive amyloid deposits and lumbar spinal canal stenosis patients with transthyretinnegative amyloid deposits. No significant difference existed in serum transthyretin concentrations of lumbar spinal canal stenosis patients with transthyretin-positive amyloid deposits and those with transthyretin-negative amyloid deposits (22.9 \pm 5.9 and $23.9 \pm 5.6 \mathrm{mg} / \mathrm{dl}$, respectively; $P=0.74$ ). In addition, mass spectrometric analysis, which can detect wild-type and mutant forms of transthyretin in serum samples, indicated that those lumbar spinal canal stenosis patients had wild-type transthyretin but no mutant transthyretin in the bloodstream.

\section{Detection of Fragmented Transthyretin in Amyloid Deposits}

To investigate the proteolytic cleavage of transthyretin in amyloid deposits, we performed immunoblotting with anti-transthyretin 50-127 antiserum. As seen in Figure 5, amyloid fibrils consisted of both full-length transthyretin and C-terminal fragmented transthyretin in lumbar spinal canal stenosis patients with transthyretin-positive amyloid deposits. 


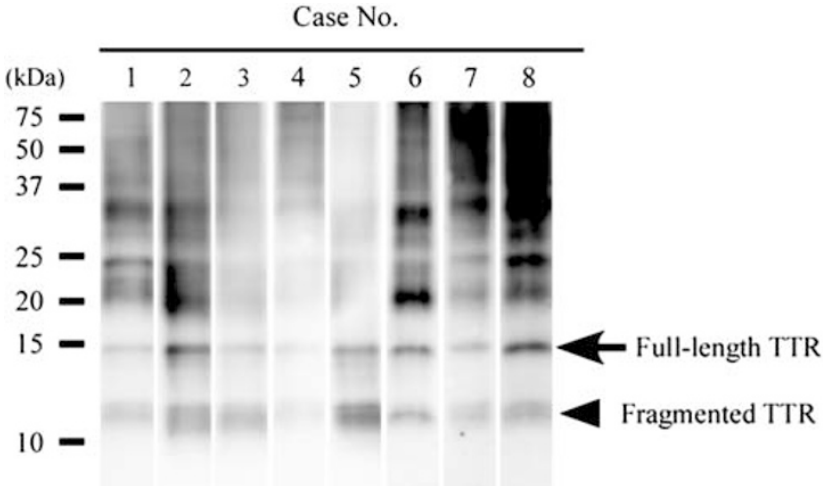

Figure 5 Immunoblotting with an antiserum against transthyretin 50-127, which can detect C-terminal transthyretin fragments in addition to full-length transthyretin. Cases 1-8 from among lumbar spinal canal stenosis patients with transthyretin-positive amyloid deposits in the ligamentum flavum were randomly selected for analysis.

\section{Discussion}

In this study, we first demonstrated that lumbar spinal canal stenosis patients often had amyloid deposits in the ligamentum flavum, which we classified into two types, transthyretin-positive and transthyretin-negative, and that transthyretin amyloid formation in the ligamentum flavum of lumbar spinal canal stenosis patients was an ageassociated phenomenon. We next established that in lumbar spinal canal stenosis patients with transthyretin-positive amyloid deposits the amount of amyloid in the ligamentum flavum was related to clinical manifestations of lumbar spinal canal stenosis, such as thickness of the ligamentum flavum and lumbar spinal segmental instability. A surprising result from this study was that all lumbar spinal canal stenosis patients had amyloid deposits in the ligamentum flavum, but the frequency of occurrence of amyloid deposits in the ligamentum flavum of lumbar disk herniation patients was limited to about $40 \%$. Amyloid deposits in the ligamentum flavum can be classified on the basis of their precursor proteins. In this study, we mainly focused on transthyretin amyloid formation in the ligamentum flavum. Transthyretin-positive amyloid deposits in the ligamentum flavum were massive and increased with the age of the lumbar spinal canal stenosis patients. Because those patients had only wild-type transthyretin in the serum, transthyretin-positive amyloid deposits in the ligamentum flavum of lumbar spinal canal stenosis patients were thought to be derived from wild-type transthyretin. These features of transthyretin amyloid deposits in the ligamentum flavum were consistent with a few previous reports that evaluated smaller numbers of lumbar spinal canal stenosis patients. ${ }^{13,14,22}$ In addition, because we discovered no relationship between the amount of transthyretin amyloid and age, we speculate that aging mainly affects the initiation of transthyretin amyloid formation in the ligamentum flavum. Because we found no symptoms that would indicate cardiac amyloidosis, which is major symptom of senile systemic amyloidosis, an age-related systemic form of amyloidosis, transthyretin-positive amyloid deposition in the ligamentum flavum of lumbar spinal canal stenosis patients may be a localized form of amyloidosis. Biochemical analysis revealed that amyloid deposits in the ligamentum flavum of lumbar spinal canal stenosis patients consisted of C-terminal transthyretin fragments, which constantly occur in amyloid deposits in senile systemic amyloidosis, in addition to full-length transthyretin. ${ }^{21,23}$ Although the roles of transthyretin fragmentation in amyloid formation remain to be clarified, there may be a common pathologic process for transthyretin amyloid formation in the ligamentum flavum of lumbar spinal canal stenosis patients and that in senile systemic amyloidosis.

The small spotty transthyretin-negative amyloid deposits were more frequently seen in younger patients, but precursor proteins of those amyloid deposits remain to be determined. To clarify features of those amyloid deposits, detailed biochemical analyses such as mass spectrometry of extracts of the deposits should be performed.

The most important finding of this study was that the amount of transthyretin amyloid in the ligamentum flavum was related to clinical manifestations of lumbar spinal canal stenosis such as the thickness of the ligamentum flavum and lumbar spinal segmental instability, because whether amyloid deposits in the ligamentum flavum would affect clinical manifestations of lumbar spinal canal stenosis had not been previously determined. This finding suggests that transthyretin amyloid deposits in the lumbar spine are related to degenerative changes in lumbar spinal canal stenosis. Dialysis-related amyloidosis, which is a complication of chronic dialysis in patients with chronic renal failure and is derived from $\beta_{2}$-microglobulin, has been well documented as causing orthopedic disorders such as destructive osteoarthropathy, carpal tunnel syndrome, and spinal involvement. ${ }^{24}$ Although we previously reported that a patient suspected of having senile systemic amyloidosis developed multifocal spinal transthyretin amyloidosis with destructive spondylosis of the lumbar spine, ${ }^{15}$ in our study here no lumbar spinal canal stenosis patients with ligamentum flavum transthyretin amyloid deposits also had destructive spondylosis. Our data here thus suggest that transthyretin amyloid deposits in the lumbar spine are related to degenerative changes and instability of the lumbar spine but rarely cause destructive changes in the lumbar spine. To clarify detailed mechanisms of transthyretin amyloid formation in the lumbar spine including causes of lumbar spinal canal stenosis or other results in lumbar spinal canal stenosis, additional studies with cell cultures and animal models are required. 
In conclusion, transthyretin amyloid deposits in the the ligamentum flavum may be related to the pathogenesis of lumbar spinal canal stenosis in elderly patients.

\section{Acknowledgments}

We are indebted to Mrs Hiroko Katsura for excellent technical assistance and Ms Judith B Gandy for providing professional English editing of the manuscript. This research was supported by Grants-inAid for Science Research from the Ministry of Education, Culture, Sports, Science, and Technology of Japan (numbers 24249036, 25870541), the Swedish Research Council, Selander's Foundation, FAMY, FAMY-Norrbotten, and Stiftelsen AMYL.

\section{Disclosure/conflict of interest}

The authors declare no conflict of interest.

\section{References}

1 Merlini G, Westermark P. The systemic amyloidoses: clearer understanding of the molecular mechanisms offers hope for more effective therapies. J Intern Med 2004;255:159-178.

2 Benson MD. Amyloidosis. In: Koopman WJ (ed) Arthritis and Allied Conditions, A Textbook of Rheumatology, 14th edn. Lippincott Williams and Wilkins: Philadelphia, PA; 2001, pp 1866-1895.

3 Sipe JD, Benson MD, Buxbaum JN, et al. Amyloid fibril protein nomenclature: 2012 recommendations from the nomenclature committee of the International Society of Amyloidosis. Amyloid 2012;19:167-170.

4 Ando Y, Nakamura M, Araki S. Transthyretin-related familial amyloidotic polyneuropathy. Arch Neurol 2005;62:1057-1062.

5 Ando Y, Ueda M. Diagnosis and therapeutic approaches to transthyretin amyloidosis. Curr Med Chem 2012;19:2312-2323.

6 Ando Y, Coelho T, Berk JL, et al. Guideline of transthyretin-related hereditary amyloidosis for clinicians. Orphanet J Rare Dis 2013;8:31.

7 Pitkänen P, Westermark P, Cornwell GG III. Senile systemic amyloidosis. Am J Pathol 1984;117:391-399.

8 Westermark P, Sletten K, Johansson B, et al. Fibril in senile systemic amyloidosis is derived from normal transthyretin. Proc Natl Acad Sci USA 1990;87: 2843-2845

9 Ueda M, Horibata Y, Shono M, et al. Clinicopathological features of senile systemic amyloidosis: an ante- and postmortem study. Mod Pathol 2011;24: 1533-1544.

10 Ihse E, Rapezzi C, Merlini G, et al. Amyloid fibrils containing fragmented ATTR may be the standard fibril composition in ATTR amyloidosis. Amyloid 2013;20:142-150.

11 D’Agostino AN, Mason MS, Quinn SF. Lumbar spinal stenosis and spondylosis associated with amyloid deposition in the ligamentum flavum. Clin Neuropathol 1992;11:147-150.

12 Honig S, Murali R. Spinal cord claudication from amyloid deposition. J Rheumatol 1992;19: 1988-1990.

13 Sueyoshi T, Ueda M, Jono $\mathrm{H}$, et al. Wild-type transthyretin-derived amyloidosis in various ligaments and tendons. Hum Pathol 2011;42:1259-1264.

14 Westermark P, Westermark GT, Suhr OB, et al. Transthyretin-derived amyloidosis: probably a common cause of lumbar spinal stenosis. Ups J Med Sci 2014;119:223-228.

15 Sueyoshi T, Ueda M, Sei A, et al. Spinal multifocal amyloidosis derived from wild-type transthyretin. Amyloid 2011;18:165-168.

16 Park JB, Chang H, Lee JK. Quantitative analysis of transforming growth factor-beta 1 in ligamentum flavum of lumbar spinal stenosis and disc herniation. Spine 2001;26:E492-E495.

17 Pitkänen MT, Manninen HI, Lindgren KA, et al. Segmental lumbar spine instability at flexionextension radiography can be predicted by conventional radiography. Clin Radiol 2002;57:632-639.

18 Dupuis PR, Yong-Hing K, Cassidy JD, et al. Radiologic diagnosis of degenerative lumbar spinal instability. Spine 1985;10:262-276.

19 Ueda M, Misumi Y, Mizuguchi M, et al. SELDI-TOF MS evaluation of variant transthyretins for diagnosis and pathogenesis of familial amyloidotic polyneuropathy. Clin Chem 2009;55:1223-1227.

20 Layfield R, Bailey K, Dineen R, et al. Application of formalin fixation to the purification of amyloid proteins. Anal Biochem 1997;253:142-144.

21 Ihse E, Suhr OB, Hellman U, et al. Variation in amount of wild-type transthyretin in different fibril and tissue types in ATTR amyloidosis. J Mol Med (Berl) 2011;89:171-180.

22 Gies U, Linke RP, Schachenmayr W. Amyloid deposits of immunohistochemically different classes in the ligamentum flavum in biopsies from patients with herniated discs or lumbar spinal stenosis. Clin Neuropathol 1996;15:54-59.

23 Bergström J, Gustavsson A, Hellman U, et al. Amyloid deposits in transthyretin-derived amyloidosis: cleaved transthyretin is associated with distinct amyloid morphology. J Pathol 2005;206:224-232.

24 Yamamoto S, Kazama JJ, Narita I, et al. Recent progress in understanding dialysis-related amyloidosis. Bone 2009;45:S39-S42.

Supplementary Information accompanies the paper on Modern Pathology website (http://www.nature.com/ modpathol) 